\title{
ON THE ERGODIC MIXING THEOREM
}

\author{
BY
}

H. A. DYE(1)

1. Introduction. An invertible measure-preserving transformation $T$ of a probability space $(\Gamma, S, m)$ is called weakly mixing if

$$
\lim \frac{1}{n} \sum_{k=0}^{n-1}\left|m\left(E \cap T^{-k} F\right)-m(E) m(F)\right|=0,
$$

or what is equivalent, if for $k$ in the complement of a set of density 0 , $m\left(E \cap T^{-k} F\right) \rightarrow m(E) m(F)$, for each pair of measurable sets $E, F$. The so-called mixing theorem of ergodic theory (see Halmos [5, p. 39]) establishes the equivalence of (1.1) and each of the following two conditions: first, that the unitary operator $U_{T}$ on $L_{2}(\Gamma, S, m)$ induced by $T$ has continuous spectrum on the subspace of $L_{2}$ orthogonal to the constant functions; and second, that the Cartesian square $T \times T$ on the product space $(\Gamma \times \Gamma, S \times S, m \times m)$ is ergodic.

This theorem appeared early in the modern development of ergodic theory, in works of von Neumann and Hopf, and unlike most of these early results, it appears to have resisted subsequent generalization. For example, no variant of the theorem seems to be known in case the measure-preserving transformation $T$ is noninvertible. In this situation the induced operator $U_{T}$ is, in general, a nonunitary isometry, to which ordinary spectral theory does not apply.

In this note we prove an abstract mixing theorem in a setting considerably more general than the above. In place of the semigroup of non-negative integers we consider an arbitrary topological semigroup $G$ which admits both a leftinvariant and a right-invariant Banach mean. This includes all abelian or compact topological semigroups, and an extensive class of discrete semigroups (see Day [1]). And, in place of the operator $U_{T}$, we consider an arbitrary weakly continuous isometric representation $U_{x}$ of $G$ on a complex Hilbert space. The operation $\lim (1 / n) \sum_{k=0}^{n-1}$ is supplanted by "almost convergence," a notion introduced by G. Lorentz [6], and in turn, the condition that $U_{T}$ have continuous spectrum is supplanted by the condition that the representation $U_{x}$ have no finite-dimensional subrepresentations. The abstract mixing theorem (proved in §3) specializes in the measure-theoretic case to a direct generalization of the classical mixing theorem (see \$4). Our proof is quite elementary, consisting of a refinement for amenable semigroups of the traditional Peter-Weyl theory.

Received by the editors January 10, 1964.

(1) This research was supported in part by a National Science Foundation grant. 
2. Technical preliminaries. Let $G$ be a topological semigroup, and $C(G)$ the sup-norm algebra of all bounded complex-valued continuous functions on $G$. By a left mean $M$ on $G$ we mean a linear functional on $C(G)$ which is positive, in the sense that $f \geqq 0$ entails $M(f) \geqq 0$, normalized so that $M(1)=1$, and leftinvariant, in the sense that $M\left({ }_{y} f\right)=M(f)$, where $\left.{ }_{y} f\right)(x)=f(y x)$, for all $f$ in $C(G)$ and all $y$ in $G$. There is a similar notion of right mean, involving the requirement that $M\left(f_{y}\right)=M(f)$, where $f_{y}(x)=f(x y)$. The semigroup $G$ is called amenable if it admits both a left and a right mean. (A discrete amenable semigroup admits a 2-sided mean, though we do not need this fact.) Invariant means are notoriously nonunique. However, as G. Lorentz has shown in [6], an invariance concept is available. For the additive semigroup $\mathrm{Z}^{+}$of non-negative integers, Lorentz proved that a bounded function $f$ is almost convergent with limit $s$, in the sense that $f$ has the same value $s$ in each invariant mean, if and only if,

$$
\lim _{n} \frac{1}{n} \sum_{k=0}^{n-1} f(k+m)=s \text { uniformly in } m .
$$

Following this lead, given an arbitrary amenable topological semigroup $G$, we will say that a function $f$ in $C(G)$ is almost convergent with limit $s$ in case $M(f)=s$ for each right mean and for each left mean $M$ on $G$.

REMARK 2.1. For general discrete semigroups, Day has introduced and characterized a concept of almost convergence [1, p. 538]. His terminology differs from ours, in that for $f$ to be almost convergent he requires only that it have the same value in each 2-sided mean.

While no completely satisfactory generalization of (2.1) for general discrete semigroups appears to be known, results in this direction, besides those of [1], can be proved using the techniques of Day and Lorentz. We cite one such result, which has a novel interpretation in terms of the theory of games. Let $G$ be a discrete amenable semigroup which is a subsemigroup of a group. Let $\alpha, \beta$ be weights on $G$, that is, non-negative functions with finite support such that $\Sigma_{x} \alpha(x)=\Sigma_{x} \beta(x)=1$, and let $f$ be a bounded real-valued function on $G$. Write $f(\alpha, \beta)=\Sigma_{x, y} \alpha(x) \beta(y) f(x y)$. We can regard $f(\alpha, \beta)$ as the pay-off in the 2-person zero-sum game in which the players play weights on $G$. In terms of game theory, this game has upper and lower values $v^{-}(f)=\operatorname{GLB}_{\beta} \operatorname{LUB}_{\alpha} f(\alpha, \beta)$ and $v_{-}(f)$ $=\operatorname{LUB}_{\alpha} \operatorname{GLB}_{\beta} f(\alpha, \beta)$. Similarly, consider the game in which $f(\alpha, \beta)$ is replaced by $f(\beta, \alpha)$ and let $w^{-}(f)$ and $w_{-}(f)$ denote its upper and lower values. Then, as one can show, in order that $f$ be almost convergent (in our sense) to limit $s$, it is necessary and sufficient that each of these games have value $s$, namely, that $v^{-}(f)=v_{-}(f)=w^{-}(f)=w_{-}(f)=s$.

Our work will involve the use of a variant of the mean ergodic theorem, which we state in the following lemma. This result is well known (see, for example, Dixmier [2, p. 233]), and we include the proof only for completeness. 
LEMMA 2.1. Let $U_{x}$ be a bounded weakly continuous representation or anti-representation of the amenable topological semigroup on a Hilbert space $\mathfrak{H}$. Let $E=E\left(U_{x}\right)$ denote the projection on the subspace $\left[f \in \mathfrak{S} \mid U_{x} f=f\right.$ for all $\left.x\right]$. Then, for each pair $f, g$ in $\mathfrak{H}$, the function $\left(U_{x} f, g\right)$ is almost convergent to a limit which, in case $U_{x}$ is isometric, is $\left(E\left(U_{x}\right) f, g\right)$.

Proof. For any mean $M$, left or right, $[f, g]=M\left(U_{x} f, g\right)$ defines a bounded form on $\mathfrak{H}$, and by a familiar lemma of Riesz, there will exist a bounded operator $Q$ on $\mathfrak{S}$ such that $M\left(U_{x} f, g\right)=(Q f, g)$. This operator $Q$ may of course depend on the particular mean. Application of a standard separation theorem (for example $[4, \mathrm{p} .417]$ ) shows that, for each vector $f, Q f$ lies in the closed convex hull of the translates $U_{x} f$ of $f$. Now we specialize a bit. Suppose that $U_{x}$ is a representation, that $M$ and $M^{\prime}$ are fixed left and right means, and that $Q$ and $Q^{\prime}$ are the corresponding operators. (In the case of an anti-representation, reverse the roles of left and right.) Then one has $Q^{\prime} U_{x}=Q^{\prime}$ and $U_{x} Q=Q$, for all $x$. Using the first of these relations, we see that $Q^{\prime} g=Q^{\prime} f$, for any convex linear combination $g$ of translates of $f$. Because $Q f$ is a limit of such linear combinations, it follows by continuity that $Q^{\prime} Q f=Q^{\prime} f, Q^{\prime} Q=Q^{\prime}$. On the other hand, using the relation $U_{x} Q f=Q f$, we see that $Q^{\prime} Q f=Q f, Q^{\prime} Q=Q$. Therefore $Q^{\prime}=Q$. This establishes the almost convergence of $\left(U_{x} f, g\right)$ to $(Q f, g)$. It is clear that $U_{x} E=E$, where $E=E\left(U_{x}\right)$. In case $U_{x}$ is isometric, so that $U_{x}^{*} U_{x}=I$, then $U_{x}^{*} E=U_{x}^{*} U_{x} E=E$, and taking adjoints, it follows that $E U_{x}=E$. The preceding argument with $E$ replacing $Q^{\prime}$ now shows that $E=Q$. This proves the lemma.

3. The abstract mixing theorem. Recall that an operator $A$ on a Hilbert space $\mathfrak{H}$ is termed Hilbert-Schmidt if, for some orthonormal base $e_{\alpha}$, $K_{A}=\Sigma_{\alpha}\left\|A e_{\alpha}\right\|^{2}<\infty$. The number $K_{A}$ will then be independent of the particular base, and one will have $K_{A}=K_{A^{*}}$.

LEMMA 3.1. Let $A$ be a Hilbert-Schmidt operator and $V$ an isometry such that $V^{*} A V=A$. Then $V A=A V$.

Proof. We can express $A$ as a linear combination $A=A_{1}+i A_{2}$ of self-adjoint Hilbert-Schmidt operators $A_{j}$, and each of these will satisfy $V^{*} A_{j} V=A_{j}$. So clearly, we may assume in what follows that $A$ itself is self-adjoint.

Let $P=V V^{*}$ be the terminal projection of $V$. Then $V A=V V^{*} A V=P A V$. We will complete the proof by showing that $P A=A$. To this end, suppose that $f$ is a vector of norm 1 which is orthogonal to the range of $V$, namely, $P f=0$. Then, the sequence $V^{n} f$ consists of mutually orthogonal vectors, and $\|A f\|=\left\|V^{*} A V f\right\| \leqq\|A V f\|=\left\|V^{*} A V^{2} f\right\| \leqq\left\|A V^{2} f\right\|$, etc. Because the $V^{n} f$ are part of an orthonormal base, $\Sigma_{n}\left\|A V^{n} f\right\|^{2}$ converges. The preceding inequalities then force $A f=0$. It follows that $A(I-P)=0$. Therefore, since $A$ is self-adjoint, $P A=A$, as asserted. 
The collection $\mathfrak{I}_{\mathrm{HS}}$ of all Hilbert-Schmidt operators on $\mathfrak{H}$ forms a self-adjoint ideal in the ring of all bounded linear operators on $\mathfrak{H}$, and $\mathfrak{I}_{\mathrm{HS}}$ becomes a Hilbert space when endowed with the inner product $(A, B)=\Sigma_{\alpha}\left(A e_{\alpha}, B e_{\alpha}\right)$. (See Dixmier [3, p. 93].)

LEMMA 3.2. Let $U_{x}$ be a weakly continuous isometric representation of the amenable topological semigroup $G$ on $\mathfrak{H}$. Then there exists a positive linear mapping $\phi$ of $\mathfrak{I}_{\mathrm{HS}}$ into itself such that $U_{x} \phi(A)=\phi(A) U_{x}$ and $M\left(A U_{x} f, U_{x} g\right)$ $=(\phi(A) f, g)$, for any right or left mean $M$ on $G$.

Proof. The given representation $U_{x}$ on $\mathfrak{H}$ induces an anti-representation $V_{x}(T)=U_{x}^{*} T U_{x}$ of $G$ on $\mathfrak{I}_{\mathrm{HS}}$. This representation is bounded:

$$
\left(V_{x}(T), V_{x}(T)\right) \leqq \sum_{\alpha}\left(T U_{x} e_{\alpha}, T U_{x} e_{\alpha}\right) \leqq \sum_{\alpha}\left(T e_{\alpha}, T e_{\alpha}\right)
$$

because the $U_{x} e_{\alpha}$ are part of an orthonormal base, showing that $\left\|V_{x}\right\| \leqq 1$. We assert that $V_{x}$ is weakly continuous. Consider first operators $T$ and $S$ of the form $T f=(f, a) a^{\prime}$ and $S f=(f, b) b^{\prime}$. One has $\left(U_{x}^{*} T U_{x} e_{\alpha}, S e_{\alpha}\right)=\left(U_{x} e_{\alpha}, a\right)\left(b, e_{\alpha}\right)\left(a^{\prime}, U_{x} b^{\prime}\right)$. Now the series $\Sigma_{\alpha}\left(U_{x} e_{\alpha}, a\right)\left(b, e_{\alpha}\right)$ converges uniformly in $x$; in fact, if $F$ is any finite subset of the set indexing the $e_{\alpha}$, then

$$
\sum_{\alpha \notin F}\left|\left(U_{x} e_{\alpha}, a\right)\left(b, e_{\alpha}\right)\right| \leqq\|a\|\left[\sum_{\alpha \notin F}\left|\left(b, e_{\alpha}\right)\right|^{2}\right]^{1 / 2},
$$

by the Schwarz inequality, and the right term can be made arbitrarily small for appropriate choice of $F$. It follows that $\left(V_{x}(T), S\right)$ is continuous in $x$ for these special operators $T, S$. This extends in turn to finite linear combinations of such operators, namely, to operators of finite rank. But operators of finite rank are dense in $\mathfrak{I}_{\mathrm{HS}}$, and the boundedness of the representation then forces the continuity of $\left(V_{x}(T), S\right)$ for arbitrary elements $T, S$ of $\mathfrak{I}_{\mathrm{HS}}$.

Applying Lemma 2.1, to the anti-representation $V_{x}$, we see that there exists a mapping $\phi(T)$ of $\mathfrak{I}_{\mathrm{HS}}$ into itself such that, for any right or left mean $M, M\left(V_{x}(T), S\right)$ $=(\phi(T), S)$. Given $f$ of norm 1 and $g$ in $\mathfrak{H}$, we imbed $f$ in an orthonormal base $e_{\alpha}$ and apply the formula just derived to the operator $S h=(h, f) g$. It results that $M\left(U_{x}^{*} T U_{x} f, g\right)=(\phi(T) f, g)$. It is clear that $\phi$ is linear and that $T \geqq 0$ forces $\phi(T) \geqq 0$. If in particular $M$ is chosen as a right mean, then we see that $U_{y}^{*} \phi(T) U_{y}=\phi(T)$. Lemma 3.1 then shows that $U_{y} \phi(T)=\phi(T) U_{y}$. The lemma is proved.

Following customary terminology, we say that a subspace $\mathfrak{M}$ of $\mathfrak{H}$ reduces the representation $U_{x}$ if both $\mathfrak{M}$ and its orthogonal complement $\mathfrak{M}^{\perp}$ are invariant under the $U_{x}$. The restriction of $U_{x}$ to a reducing subspace is called a subrepresentation of $U_{x}$. When $U_{x}$ is isometric, it is elementary that any finite dimensional invariant subspace $\mathfrak{M}$ reduces the representation; in fact, if $f \in \mathfrak{M}, g \in \mathfrak{M}{ }^{\perp}$, then we can find an $h$ in $\mathfrak{M}$ such that $U_{x} h=f$, since $U_{x}$ must map $\mathfrak{M}$ onto itself, and therefore $\left(f, U_{x} g\right)=\left(U_{x} h, U_{x} g\right)=(h, g)=0$, showing that $\mathfrak{M}^{\perp}$ is also invariant. 
It follows that any finite dimensional isometric (viz. unitary) representation of the semigroup $G$ is a direct sum of irreducible subrepresentations. Proceeding now with a weakly continuous isometric representation $U_{x}$ of the amenable semigroup $G$ on $\mathfrak{H}$, we make the key application of Lemma 3.2.

Lemma 3.3. Given $f$ in $\mathfrak{H}$, in order that there exist a finite dimensional projection $P$ commuting with the $U_{x}$ and such that $P f \neq 0$, it is necessary and sufficient that there exist an $A$ in $\mathfrak{I}_{\mathrm{HS}}$ such that $\phi(A) f \neq 0$.

Proof. If $P$ is a finite dimensional projection commuting with the $U_{x}$, then it is obvious that $\phi(P)=P$. Conversely, suppose that there exists a HilbertSchmidt $A$ such that $\phi(A) f \neq 0$. Because $\phi$ conserves adjoints, we can assume that $A$ and so $\phi(A)$ is self-adjoint. By spectral theory for compact operators, there exists a finite dimensional projection $P$ which is a uniform limit of polynomials in $\phi(A)$ such that $P f \neq 0$. Because $\phi(A)$ commutes with the $U_{x}$, the same must hold for $P$. The lemma is proved.

Let $\mathfrak{N}$ be the subspace of $\mathfrak{H}$ consisting of all vectors $f$ such that $\left|\left(U_{x} f, g\right)\right|$ is almost convergent to 0 for each $g$ in $\mathfrak{H}$. (The lemma to follow will contain the information that if, for a particular left or right mean, $M\left(\left|\left(U_{x} f, g\right)\right|\right)=0$ for each $g$, then already $f$ lies in $\mathfrak{N}$.)

LEMMA 3.4. $\mathfrak{N}$ reduces the representation $U_{x}$, and the restriction of $U_{x}$ to $\mathfrak{N}$ has no finite dimensional subrepresentations. On the other hand, the restriction of $U_{x}$ to $\mathfrak{N}^{\perp}$ is unitary and is a direct sum of irreducible weakly continuous finite dimensional subrepresentations.

Proof. Let $P_{\alpha}$ be a maximal collection of mutually orthogonal nonzero finite dimensional projections, each commuting with the representation, and set $P=\sum_{\alpha} P_{\alpha}$. Clearly $P U_{x}=U_{x} P$, and since $U_{x} U_{x}^{*} P_{\alpha}=P_{x}$ for each $\alpha$, by finite dimensionality, it follows also that $U_{x} U_{x}^{*} P=P$, so that $U_{x}$ is unitary on the range of $P$. Now $U_{x}$ restricted to the range of $I-P$ has no finite dimensional subrepresentations. In fact, were there to exist a finite dimensional projection $Q$ commuting with the representation and such that $Q(I-P) \neq 0$, then $(I-P) Q(I-P)$ is a nonzero self-adjoint compact operator commuting with the representation, and so we can clearly find a nonzero finite dimensional projection $R$ commuting with the representation and orthogonal to all $P_{\alpha}$, contradicting the maximality of that collection.

Application of Lemma 3.3 now shows that the range of $I-P$ consists precisely of those vectors $f$ such that $\phi(A) f=0$, for all $A$ in $\mathfrak{I}_{\mathrm{HS}}$. We will complete the proof by showing that the range of $I-P$ is the same as $\mathfrak{R}$. To see this, select a particular right or left mean $M$ and a vector $f$ such that $M\left(\left|\left(U_{x} f, g\right)\right|\right)=0$ for all $g$. For each operator $A$ of the form $A f=(f, g) g^{\prime}$, one has $|(\phi(A) f, h)| \leqq M\left(\left|\left(A U_{x} f, U_{x} h\right)\right|\right)$ $=M\left(\left|\left(U_{x} f, g\right)\left(g^{\prime}, U_{x} h\right)\right|\right) \leqq\left\|g^{\prime}\right\|\|h\| M\left(\left|\left(U_{x} f, g\right)\right|\right)=0$, so $\phi(A) f=0$. On the other hand, if $\phi(A) f=0$ for all $A$ in $\mathfrak{I}_{\mathrm{HS}}$, and if we take $A$ to be the operator 
$A f=(f, g) g$, then $0=(\phi(A) f, f)=M\left(A U_{x} f, U_{x} f\right)=M\left(\left|\left(U_{x} f, g\right)\right|^{2}\right)$. By the Schwarz inequality, it follows that $M\left(\left|\left(U_{x} f, g\right)\right|\right)=0$ for each $g$, and since $M$ is arbitrary, that $\left|\left(U_{x} f, g\right)\right|$ is almost convergent to 0 .

Let $J$ be a conjugation of $\mathfrak{H}$, that is, a conjugate linear mapping of $\mathfrak{H}$ into itself such that $J^{2}=I$ and $(J f, J g)=(g, f)$. If $U_{x}$ is a weakly continuous isometric representation of $G$ on $\mathfrak{G}$, then the same is true of the representation $U_{x}^{J}=J U_{x} J$, as well as of the Kronecker product $U_{x} \otimes U_{x}^{J}$ on $\mathfrak{H} \otimes \mathfrak{S}$ of the representations $U_{x}$ and $U_{x}^{J}$.

THEOREM 1. Let $U_{x}$ be a weakly continuous isometric representation of the amenable topological semigroup $G$ on a Hilbert space $\mathfrak{H}$, and let $J$ be a conjugation of $\mathfrak{H}$. Then the following conditions are equivalent:

(i) $\left|\left(U_{x} f, g\right)\right|$ is almost convergent to 0 , for each pair $f, g$ in $\mathfrak{S}$;

(ii) $U_{x}$ has no finite dimensional subrepresentations; and

(iii) $E\left(U_{x} \otimes U_{x}^{J}\right)=0$.

Proof. (The notation in (iii) is that of Lemma 2.1.) Lemma 5 shows that $\mathfrak{N}=\mathfrak{H}$ if and only if $U_{x}$ has no finite dimensional subrepresentations, and so the equivalence of (i) and (ii) follows. We will show that (i) and (iii) are equivalent. Let $M$ be any right or left mean on $G$. Then if $E\left(U_{x} \otimes U_{x}^{J}\right)=0$, we see by Lemma 2.1 that $0=M\left(\left(U_{x} \otimes U_{x}^{J}(f \otimes J f), g \otimes J g\right)\right)=M\left(\left(U_{x} f, g\right)\left(J U_{x} f, J g\right)\right)$ $=M\left(\left|\left(U_{x} f, g\right)\right|^{2}\right)$, and as before it follows that $M\left(\left|\left(U_{x} f, g\right)\right|\right)=0$ for all $f$ and $g$. Conversely, it is easy to see that if $M\left(\left|\left(U_{x} f, g\right)\right|\right)=0$ for all $f, g$, then $M\left(\left(U_{x} \otimes U_{x}^{\mathrm{J}}\left(f \otimes f^{\prime}\right), g \otimes g^{\prime}\right)\right)=0$ for all $f, f^{\prime}, g, g^{\prime}$, and linearity and continuity imply that $M\left(\left(U_{x} \otimes U_{x}^{J} u, v\right)\right)=0$ for all $u, v$ in $\mathfrak{H} \otimes \mathfrak{H}$, so that $E\left(U_{x} \otimes U_{x}^{J}\right)=0$.

EXAMPLE. There exist minimally almost periodic discrete amenable groups, that is, discrete amenable groups having no nontrivial finite dimensional unitary representations. For any unitary representation of such a group, the various conditions of the theorem then apply. We give a simple example of such a group.

Let $F$ be an infinite field of characteristic 2 which is the union of a tower of finite subfields, and let $G$ be the group of all $2 \times 2$ matrices

$$
\left(\begin{array}{ll}
a & b \\
c & d
\end{array}\right)
$$

over $F$ with determinant $a d+b c=1$. Evidently, $G$ is a locally finite group, that is, every finitely generated subgroup is finite, and any such group is known to be amenable [1, p. 517]. Let $H$ be the subgroup of $G$ consisting of all matrices

$$
\left(\begin{array}{ll}
1 & b \\
0 & 1
\end{array}\right)
$$

$b$ ranging over $F . H$ and the transposed subgroup $H^{t}$ together generate $G$; in 
fact, a simple computation shows that each element of $G$ can be expressed as the product of four elements chosen from $H$ and $H^{t}$. For each $x \neq 0$,

$$
\left(\begin{array}{ll}
x & 0 \\
0 & x^{-1}
\end{array}\right)\left(\begin{array}{ll}
1 & b \\
0 & 1
\end{array}\right)\left(\begin{array}{ll}
x & 0 \\
0 & x^{-1}
\end{array}\right)^{-1}=\left(\begin{array}{cc}
1 & x^{2} b \\
0 & 1
\end{array}\right),
$$

and since $x \rightarrow x^{2}$ is an automorphism of $F$, the automorphisms of $H$ arising by (3.2) as $x$ varies have two orbits in $H$, the identity, and the complement in $H$ of the identity.

Let $U_{g}$ be a finite dimensional unitary representation of $G$, and let $U_{b}$ denote the unitary matrix representing the element (3.1) of $H$. The preceding discussion shows that either $U_{b}=I$ for all $b$ or $U_{b}$ is faithful on $H$. We argue, the second case cannot occur. Suppose it did. The closure in the uniform topology of the $U_{b}$ 's is a compact group, and the identity $I$ cannot be an isolated point in this group; for if it were, the compact group and a fortiori the original group of $U_{b}$ 's would be finite. It follows that there exists a sequence $b_{n} \neq 0$ such that $U_{b \text {.. }}$ converges uniformly to $I$. Then Trace $U_{b_{n}} \rightarrow$ Trace $I$. But since all the $U_{b}(b \neq 0)$ have the same trace, in view of (3.2), it follows that. Trace $U_{b}=$ Trace $I$ for all $b$. But any unitary matrix $U$ with Trace $U=$ Trace $I$ is necessarily $I$, and we have a contradiction.

Therefore, $H$ lies in the kernel of the representation $U_{g}$. Likewise $H^{t}$ lies in the kernel of $U_{g}$, and since $H$ and $H^{t}$ generate $G$, the representation is trivial on $G$.

4. Application to ergodic theory. The abstract mixing theorem just proved leads directly to a generalization of the ergodic mixing theorem, which we now indicate. Let $(\Gamma, S, m)$ be a finite measure space, with the normalization $m(\Gamma)=1$, and let $G$ be an amenable topological semigroup. Suppose to each element $x$ of $G$ there is assigned a measure-preserving transformation $T_{x}$ of $(\Gamma, S, m)$ (meaning $T_{x}^{-1}$ conserves measurability and measure) in such a way that $T_{x y}=T_{y} T_{x}$ and $x \rightarrow m\left(E \cap T_{x}^{-1} F\right)$ is continuous. We then call $T_{x}$ a continuous measure-preserving anti-representation of $G$. This representation is called ergodic if, for a measurable set $E, T_{x}^{-1} E \subseteq E$ for all $x$ implies $E$ or its complement has measure $0 . T_{x}$ induces a weakly continuous isometric representation $U_{x}$ of $G$ on the Hilbert space $\Re=L_{2}(\Gamma, S, m)$ via $U_{x} f(\gamma)=f\left(T_{x} \gamma\right)$. Lemma 2.1 shows that, for each $f$ in $\Omega, U_{x} f$ is weakly almost convergent to the projection $E\left(U_{x}\right) f$ of $f$ on the subspace $\left[f \in \Omega \mid U_{x} f=f\right.$ for all $\left.x\right]$. As is well known, the semigroup $T_{x}$ is ergodic if and only if $E\left(U_{x}\right)$ coincides with the projection $E_{[1]}$ on the subspace [1] of constant functions.

CoROllary 1. Let $T_{x}$ be a continuous measure-preserving anti-representation of an amenable topological semigroup on a finite measure space $(\Gamma, S, m)$, and let $U_{x}$ be the induced weakly continuous isometric representation of $G$ on $\boldsymbol{\Omega}=L_{2}(\Gamma, S, m)$. Then the following conditions are equivalent:

(i) $\left|m\left(E \cap T_{x}^{-1} F\right)-m(E) m(F)\right|$ is almost convergent to 0 , for each pair of measurable sets $E$ and $F$; 
(ii) The only finite dimensional subrepresentation of $U_{x}$ is its restriction (the identity) to the subspace of constant functions; and

(iii) The semigroup of product transformations $T_{x} \times T_{x}$ on the product measure space $(\Gamma \times \Gamma, S \times S, m \times m)$ is ergodic.

Proof. Let $J$ be the operation on $\Re$ of ordinary complex conjugation: $J f=\bar{f}$. Then clearly $U_{x}^{J}=U_{x}$. The projection $E=E_{[1]}$ commutes with $U_{x}$, and so if $\mathfrak{H}$ denotes the range of $I-E$, then $\mathfrak{S}$ reduces the representation $U_{x}$. Let $V_{x}$ denote the restriction of $U_{x}$ to $\mathfrak{H}$. Obviously $\mathfrak{S}$ is $J$-invariant. We can apply Theorem 1 to the weakly continuous isometric representation $V_{x}$ of $G$ on $\mathfrak{H}$, taking for the conjugation the $J$ just defined. We consider the three conditions of Theorem 1 . For $f, g$ in $\Omega$, we have $\left(U_{x} f, g\right)=\left(U_{x} E f, E g\right)+\left(U_{x}(I-E), f(I-E) g\right)=(f, 1)(1, g)$ $+\left(V_{x}(I-E) f,(I-E) g\right)$, so $\left|\left(U_{x} f, g\right)-(f, 1)(1, g)\right|=\left|\left(V_{x}(I-E) f,(I-E) g\right)\right|$. Condition (i) of Theorem 1 provides that the right side here be almost convergent to 0 for all $f, g$. In the present situation, by continuity and linearity, this is therefore equivalent to condition (i) of the corollary. (Bear in mind that $m(\Gamma)=1$.) Condition (ii) of the theorem provides that $V_{x}$ have no finite dimensional subrepresentations on $\mathfrak{H}$. Now if $F$ is a nonzero finite dimensional projection on $\mathfrak{R}$ commuting with $U_{x}$, and, if $U_{x}(I-E)$ has no finite dimensional subrepresentations, then it is trivial that $F(I-E)=0, F \leqq E$, whence, $F=E$. Therefore condition (ii) of the corollary is equivalent to condition (ii) of the theorem. We turn finally to (iii). $T_{x} \times T_{x}$ is easily seen to be a continuous measure-preserving anti-representation of $G$ on the product measure space, and it is trivial that we can identify the tensor product $\Omega \otimes \Re$ with $L_{2}$ of the product space, $\mathfrak{H} \otimes \mathfrak{H}$ being identified in the process with the orthogonal complement of the subspace of constant functions. Now, by the remark preceding the corollary, the ergodicity of $T_{x} \times T_{x}$ is equivalent to the statement that $E\left(U_{x} \otimes U_{x}\right)$ is the projection on the subspace of constant functions. The equivalence of the conditions (iii) follows readily.

Theorem 1 is of course applicable to representations $U_{x}$ induced by continuous measure-preserving anti-representations of $G$ on (infinite) $\sigma$-finite measure spaces. The corollary, however, breaks down.

\section{REFERENCES}

1. M. Day, Amenable semigroups, Illinois J. Math. 1 (1957), 509-544.

2. J. Dixmier, Les moyennes invariantes dansles semi-groupes et leurs applications, Acta Sci. Math. (Szeged) 12 (1950), 213-227.

3. - Les algèbres d'opérateurs dans l'espace Hilbertien, Gauthier-Villars, Paris, 1957.

4. N. Dunford and J. Schwartz, Linear operators, Vol. I, Interscience, New York, 1958.

5. P. Halmos, Ergodic theory, Publ. Math. Soc. Japan, No. 3, Math. Soc. Japan, Tokyo, 1956.

6. G. Lorentz, A contribution to the theory of divergent sequences, Acta Math. 80 (1948), 167-190.

UNIVERSITY OF CALIFORNIA, Los ANgeles, California 